\title{
Subsurface Corrosion Imaging System Based on LASER Generated Acoustic (LGA)
}

\author{
Andreas Setiawan", Gede Bayu Suparta*, Mitrayana*, Waskito Nugroho* \\ "Department of Physics, Satya Wacana Christian University, Jl. Diponegoro 52-60, Salatiga, 50711, Indonesia \\ E-mail: andreas.setiawan@staff.uksw.edu
}

*Department of Physics, Universitas Gadjah Mada, Sekip Utara Bulaksumur, Yogyakarta, 55281, Indonesia

E-mail: gbsuparta@ugm.ac.id, mitrayana@ugm.ac.id,wnugroho2000@gmail.com

\begin{abstract}
Corrosion process might occur over or beneath the surface of a material. Under-the-surface corrosion is more dangerous than other types as it is visually hidden. This research develops an imaging system to detect crevices resulting from subsurface corrosion. The system is built with a laser-generated acoustic (LGA) method, - using photoacoustic phenomena to generate acoustic waves from a laser and material interaction process, to construct a subsurface image of a metal. Though optical actuator is used, deeper penetration is achieved in the acoustic wave sensing, causing a change of intensity of the waves as they pass corrosion crevices. Measurement of the acoustic wave's intensity is used to construct LGA image illustrating the subsurface condition of an object. There are three main components of the device, which are a laser, a microphone and a data processor. The laser beam is modulated and exposed to an object to create thermal contraction. The microphone records acoustic waves generated in the process and the data processor analyses the result. Comprehensive measurement is done over the entire surface of the object to create 2-dimensional images. This research reports an experimental result of the imaging system on an object with subsurface corrosion crevices. LGA's images produced showed reasonably clear evolution process of the corrosion. In the experiment, the crevices emerge at $230 \mu \mathrm{m}$ depth were detected in the first 5 hours of the corrosion process. An advance analysis showed that the LGA images produced were able to show the evolution process of the shape of the crevices during corrosion. A mathematical model, based on acoustic transmission intensity equation, is designed to further examine the LGA image resulting in a curve which fits the measurement result with MAPE $12 \%$ difference.
\end{abstract}

Keywords - laser-generated acoustic; photoacoustic imaging; subsurface corrosion

\section{INTRODUCTION}

Corrosion is a degradation process of a material due to chemical reaction with its environment. Various studies have been reported by many researchers on the characteristic of corrosion to better mitigate its effect [1]-[5]. Corrosion may occur on the surface of an object or within it depending on the exposed parts to corrosive materials. However, corrosion beneath the surface has a higher risk of destruction as the initial symptoms of the damage are visually veiled. If it is not stopped in the early stage, eventually corrosion will spread throughout the object causing more massive destruction.

Subsurface is a term commonly used for areas of corrosion that are invisible, for example, corrosion of metal beneath layers of paints which is called corrosion under the paint (CUP) [6]. Radio wave, a non-contact detection method is generally used in the detection of CUP as it is superficial. Terahertz NDE method, by using radio waves, is reported to detect roughness level of CUP up to $12.5 \mu \mathrm{m}$ [7].
On the other hand, by using near-field millimeter-wave Kaband (26.5-40 GHz) dan V-band $(50-75 \mathrm{GHz})$ probes, it detected artificial CUP in a range of $150 \mu \mathrm{m}$ to $500 \mu \mathrm{m}$ [8]. Another type of subsurface is corrosion under insulation (CUI) which occurs on the surface of an object covered with an insulation material [9]. In general, the cover refers to thermal insulation used to reduce energy lost in a heating or cooling system [10]. Leaked insulation might cause liquid to flow in and trapped between the layers. This humid condition progressively will lead to corrosion on the surface of an object under the insulation layer. CUI has a significant impact on most industries as they use insulation method to increase their efficiency [11].

Basically, CUP and CUI occur on metal structures where their surfaces are covered by other materials. However, subsurface corrosion might happen directly inside the metal body, which is slightly different from the other two subsurface corrosions. A contaminant particle trapped inside a material or spreading of oxidation from other parts such as pitting or crevice corrosion might trigger this type of 
corrosion [9], [12]. This corrosion occurs as a result of abundant exposure of corrosive materials on a certain area which produces holes on the surface of an object. The holes lead to pits and eventually creates crevices over continues contact. Subsurface and undercutting corrosions are the examples of the evolution model of pits to crevices [13]. Sometimes, the tip of pits location is difficult to reach, and the crevices propagate inside the material. As the indicators of corrosion are hidden inside the body, the object's surface is intact while the destruction is happening within. These conditions require a different method of detection from CUI and CUP as the corrosion symptoms are hidden, even after removing the insulation from the surface.

This research is aimed to develop an imaging system to detect subsurface crevice corrosion inside a material by using acoustic wave interaction as results of the modulated laser, which is called Laser Generated Acoustic (LGA). This interaction is suitable for the gaps to cause an alteration in acoustic transmission coefficient. Therefore the intensity of the waves will shift as they pass through the crevice. Accordingly, a low scattering level with depth penetration ability of acoustic waves is used to construct an image of the subsurface structure of an object including the crevices. To avoid mechanic coupling problem commonly experienced in a conventional ultrasonic system with piezoelectric PZT transducer, this research uses an LGA as an actuator as it uses photoacoustic phenomena to produce acoustic waves directly on the surface of an object [14]. Thus, the mismatching impedance can be avoided as wave's propagation into the object doesn't need any other medium.

A complete imaging system is shown in Fig. 1, which consists of three main components, beam source, acoustic sensor and a data processor. A continuous laser diode is used as an exciter source as it is cheap and compact [15-16], while the acoustic sensor is a standard audio microphone working in audible frequency to eliminate the need of highspeed detector (ultrasonic) in the data processing [17]. Data collection is done by using an $\mathrm{x}-\mathrm{y}$ stage driver to measure each point on the surface area to create two-dimensional data array which is used to construct an LGA image of the object.

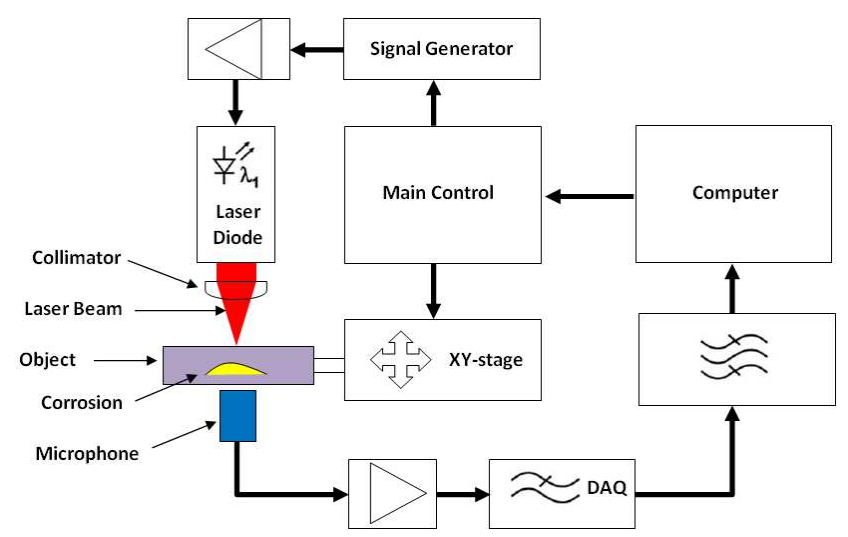

Fig. 1 LGA imaging system diagram consists of three main components; a laser diode, a microphone and a data processor. The Laser Generated Acoustic process occurs when there is a laser - object interaction. Acoustic signals generated in the process are recorded by the microphone on each point on the surface to create a two-dimension LGA image

The result of experiments on subsurface corrosion imaging system of carbon steel is described in the last part of this report. A line profile analysis of the LGA image showed a detailed evolution process of crevice corrosion at 0.23 to $0.73 \mathrm{~mm}$ depth. The system is able to examine the development of crevice in the first 5 hours after corrosion process begins including the shape of the crevice. A mathematical model of acoustic transmission of the LGA is designed, and the calculation shows a good fitting curve of the measurement with MAPE difference level about $12.5 \%$.

\section{MATERIAL AND METHOD}

\section{A. Material}

Artificial corrosion is introduced on a surface of an object to create research sample which is done by covering a carbon steel plate with paint while leaving some part of it open for exposure to corrosive materials. The sample is submerged in a concentrated corrosive solution Ferric Chloride $\left(\mathrm{FeCL}_{3}\right)$ for some time. The illustration of sample making on an object is shown in Fig. 2. An image of the sample before initiation of corrosion is taken as a reference which will be compared to images after immersion. Images are repeatedly taken until crevice is clearly detected. The subsurface structure is projected from the opposite side of the surface with the corrosion.

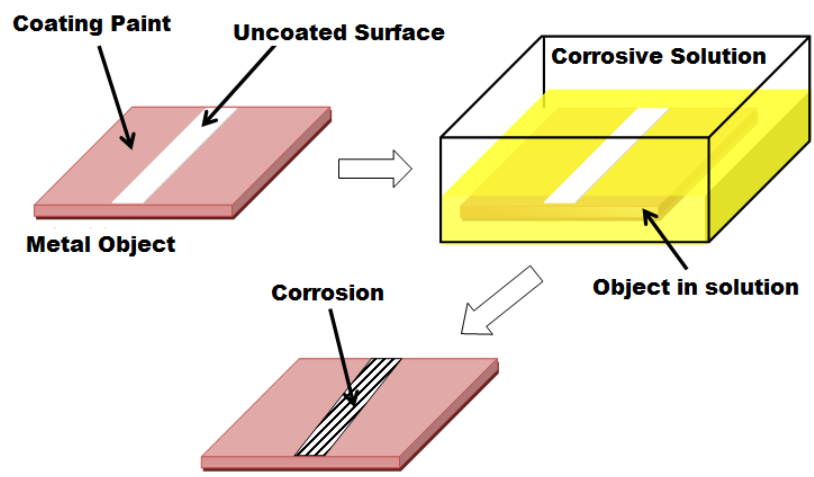

Fig. 2 The sample is made of a steel carbon plate covered with protective paint with some part is exposed to a concentrated corrosive solution Ferric Chloride $\left(\mathrm{FeCl}_{3}\right)$. To construct a subsurface image of the corrosion, imaging process is done on the other side of the surface. The process is repeated to create images of the evolution of corrosion

\section{B. Laser Generated Acoustic}

Photoacoustic model is designed by coupling mechanic wave's equation and thermal diffusion on a surface material exposed to a laser beam [18]. The laser beam, in the form of short pulses or modulated waves, is used as the source of energy to thermally activate the material. The interaction between the beam and material causes heating on the exposed points on the surface material. Relationship connecting volume $V$ and pressure $p$ on a surface heated to temperature $\Theta$ at $r$ position is expressed as

$$
\frac{d V}{V}=-\kappa p(r)+\beta T \Theta(r) .
$$

From the equation, $\beta$ is thermal expansion coefficient of volume, while $\kappa$ is isothermal compressibility which is related to the material parameter in the form as follow

$$
\kappa=\frac{C_{p}}{\rho v_{s}^{2} C_{V}} .
$$


Terms $\rho$ and $v_{s}$ are density and speed of sound respectively. $C_{P}$ is heat capacity at constant pressure, and $C_{V}$ is heat capacity at constant volume. For solid objects, both terms, $C_{P}$ and $C_{V}$, will have similar values that cancel each other out. As the laser beam is modulated quickly, heating process has no significant effect on the volume. Thus its change is negligible in the equation. Using Equation 1, the acoustic pressure produced can be determined by [19]

$$
p_{0}(r)=\frac{\beta \Theta(r)}{\kappa} .
$$

Assumed that all energy of laser beam pulse effectively transformed into heat, while other non-thermal energy lost is insignificant, the temperature increase of the material is given by the following

$$
\Theta=\frac{A_{e}}{\rho C_{V}}
$$

with $A_{e}$ is optical absorption per unit volume (or optical energy density deposition). From Equation 3 and 4, acoustic pressure generated by the photoacoustic process is given below [19]

$$
p_{0}=\frac{\beta}{\kappa \rho C_{V}} A_{e} .
$$

The term $\beta / \kappa \rho C_{V}$ is called Gruneisen parameter which gives the empirical approach value for optical-mechanic conversion of certain material. Some empirical approaches of these parameters are discussed in a research report written by Vocadlo et al. [20].

\section{Acoustic Transmission}

When acoustic waves go through one medium to another, at the border of the two mediums, the waves will be reflected and transmitted. The ratio of the waves' intensity and pressure amplitude depend on the characteristic of acoustic impedance, the speed of sound in each medium and incident angle of the wave [21]. Fig. 3 illustrates incident, reflected and transmitted waves with an angle $\theta_{i}, \theta_{r}$ and $\theta_{t}$ respectively.

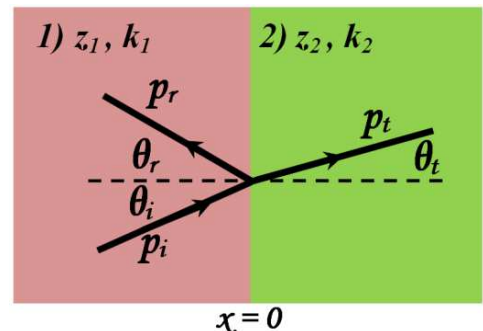

Fig. 3 Reflected and transmitted waves are generated when acoustic waves go through the border of medium 1 and 2 which have different characteristics. At $x=0$, continuity condition applied for both pressure and speed of wave. Thus transmission intensity can be calculated

By applying pressure continuity on $x=0$, wave equation for xy plane can be derived as

$$
\begin{aligned}
& P_{i} e^{j\left(\omega t-k_{1} x \cos \theta_{i}-k_{1} y \sin \theta_{i}\right)}+P_{r} e^{j\left(\omega t+k_{1} x \cos \theta_{r}-k_{1} y \sin \theta_{r}\right)} \\
& =P_{t} e^{j\left(\omega t-k_{2} x \cos \theta_{t}-k_{2} y \sin \theta_{t}\right)}
\end{aligned}
$$

Another quantity to justify is the continuity condition of velocity vector, with $u_{j}$ as speed component of each wave; the equation can be rewritten as

$$
u_{i} \cos \theta_{i}+u_{r} \cos \theta_{r}=u_{t} \cos \theta_{t} .
$$

The relationship between velocity and acoustic impedance $u_{j}= \pm p / z$ can be used to link Equation 1 and 2. In the condition where the angles $\theta_{i}=\theta_{r}, 1+R=T$ and $T_{I}=\left(z_{1} / z_{2}\right)|T|^{2} \quad ;$ the intensity transmission coefficient can be calculated by the following equation

$$
T_{I}=\frac{4 z_{2} / z_{1}}{\left(z_{2} / z_{1}+1\right)^{2}}
$$

\section{RESULT AND DISCUSSION}

\section{A. Acoustic Generating Simulation}

Equation 5, acoustic pressure generated in the photoacoustic process will be the initiation source to stimulate acoustic wave's propagation. In general, the equation of wave function with an external source $\phi_{\text {ext }}$ is written as [18]

$$
\left(\nabla^{2}-\frac{1}{v_{s}^{2}} \frac{\partial^{2}}{\partial t^{2}}\right) p=\phi_{e x t}
$$

In two-dimension, the Equation 9 can be rewritten as

$$
\frac{\partial^{2} p}{\partial x^{2}}+\frac{\partial^{2} p}{\partial y^{2}}=\frac{1}{v_{s}^{2}} \frac{\partial^{2} p}{\partial t^{2}}-\phi_{e x t} .
$$

Accordingly, if the laser beam has a temporal function, $\delta(x) \delta(y) \delta(t) \quad$ while the external source, $p_{0} \delta(x) \delta(y) \delta(t)$ will produce coupled two-dimension wave function in form of

$$
\frac{\partial^{2} p}{\partial x^{2}}+\frac{\partial^{2} p}{\partial y^{2}}=\frac{1}{c^{2}} \frac{\partial^{2} p}{\partial t^{2}}-p_{0} \delta(x) \delta(y) \delta(t) .
$$

Calculation of Green function can be used to solve Equation 11 [22]. By using Laplace transformation in the final calculation, the solution is given as follow

$$
p(x, y, t)=\frac{v_{s} p_{0}}{2 \pi} \frac{H\left(v_{s} t-r\right)}{\sqrt{\left(v_{s} t\right)^{2}-r^{2}}} .
$$

The wave propagation equation can be determined by placing few parameters in Equation 12 for the calculation below

$$
p(x, y, t)=\frac{\beta A_{e}}{2 \pi v_{s} \kappa \rho C_{V}} \begin{cases}0 & : v_{s} t<r \\ 1 / \sqrt{\left(v_{s} t\right)^{2}-r^{2}} & : v_{s} t>r\end{cases}
$$


The Equation 13 illustrates the spreading pattern of acoustic waves emerging after the object was exposed to the laser. Acoustic waves appeared with the development of instantaneous initial pressure after the material contracts.

The simulation process is run to produce an illustration of acoustic propagation pattern by using a carbon steel plate (10 $\mathrm{x} 10 \mathrm{~cm})$ as virtual object and speed of acoustic wave of $6000 \mathrm{~m} / \mathrm{s}$. Short pulse laser beam is directed precisely in the middle of the object, and the acoustic waves generated in this procedure are recorded every $3 \mu \mathrm{s}$. The calculation result of Equation 1 at interval time of 1 to $10 \mu \mathrm{s}$ is presented in Fig. 4. From the images, it is shown that a moment after the laser was exposed, a strong peak of initial pressure emerged at $1 \mu \mathrm{s}$. At this moment, the acoustic waves are generated directly on the object's surface by LGA process. The initial pressure is the initiator of the waves' propagation on the next phase which can be seen spreading in a circular pattern towards the edge of the object at $10 \mu \mathrm{s}$.
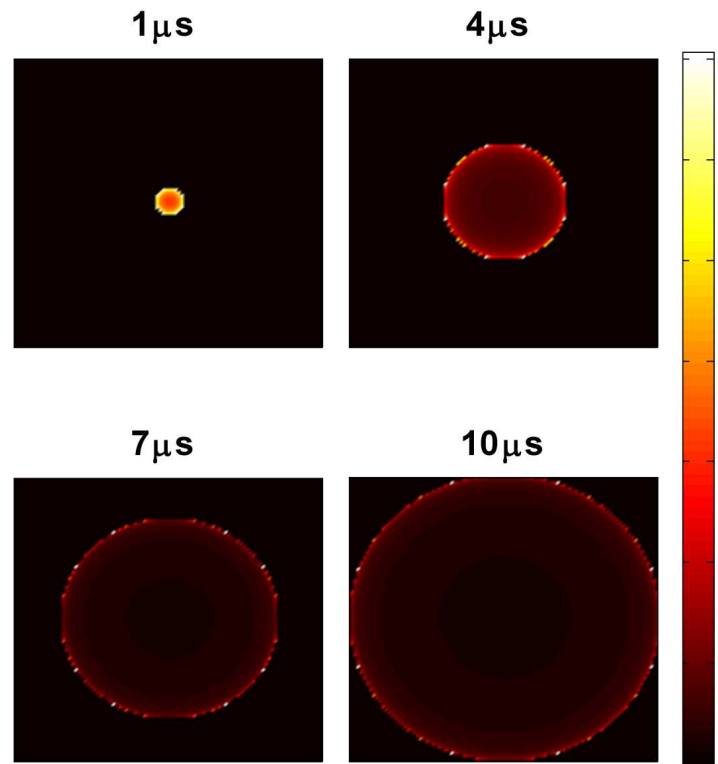

Fig. 4 LGA simulation result on a carbon steel plate $(10 \times 10 \mathrm{~cm})$ with acoustic wave's speed $6000 \mathrm{~m} / \mathrm{s}$. A moment after LGA interaction, at $1 \mu \mathrm{s}$, strong pressure emerged as initial trigger wavefronts spreading out the source in a circular pattern. At $10 \mu \mathrm{s}$, as the wavefronts reached the edge of the object, the amplitude decreased to $50 \%$.

\section{B. LGA Signal Measurement}

LGA signal is measured by using audio recorder device PC Real Time Audio Spectrum Analyser (RTA 168) from Virtins Technology with main components of Measurement Microphone (ECM999, bandwidth 20-20 kHz) and XLR-toUSB Sound Card (sampling rate $48 \mathrm{kHz}, 16$ bit). Fig. 5 shows the arrangement of the devices. A laser was exposed perpendicularly to a carbon foil placed over a microphone layered with ultrasonic gel (Ultrasound Transmission Gel, General Care Ltd.) to create stronger bonding.

The laser is modulated at three different frequencies, $1.7 \times 10^{4}, 1.4 \times 10^{4}$ and $1.3 \times 10^{4} \mathrm{~Hz}$ and each is recorded for 30 seconds. Spectrum curve is created with Fourier transformation by using the data collected. Observation of the spectrum gives a better result as the recorded data are mixed with background signal and noise during the measurement. Investigating the frequency helps to separate the LGA signal from the unwanted disruption. The measurement result of the three spectrum curves is shown in Fig. 6. The graph shows that there is peaks signal with a frequency which is relevant to its modulating laser. This means that the microphone detects the acoustic waves produced by a photoacoustic process using LGA. The amplitude of the acoustic is significantly higher than the noise that makes it easy to isolate the proposed signal. Measurement result shows average amplitude is $-63 \mathrm{~dB}$ with the lowest Signal to Noise Ratio (SNR) is 21 as calculated from modulation curve with frequency $1.3 \times 10^{4} \mathrm{~Hz}$ with an assumption that the microphone response is fixed on $-70 \mathrm{~dB}$. At a lower frequency, there is a significant increase of disruption caused by preamp noise which tends to be stronger [23]. Thus, in this measurement, the LGA signal modulation in a range of thousands $\mathrm{Hz}$ is effectively used as it produces significantly high amplitude.

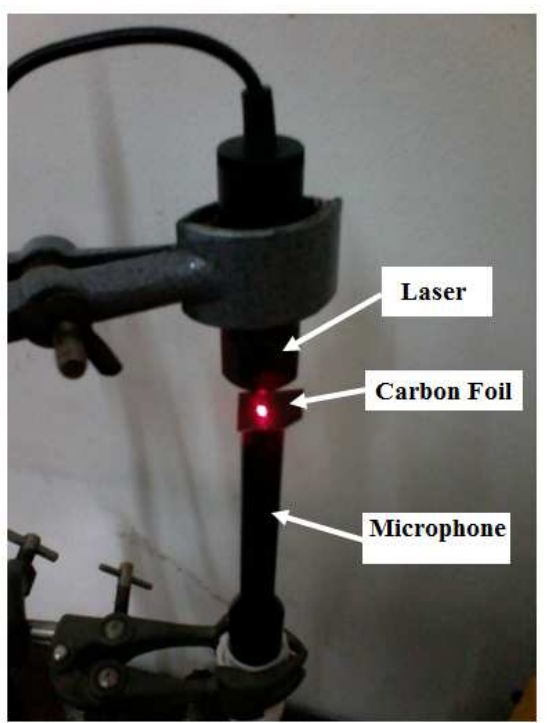

Fig. 5 Experimental Setup for Measuring Photoacoustic Signals on a Carbon foil

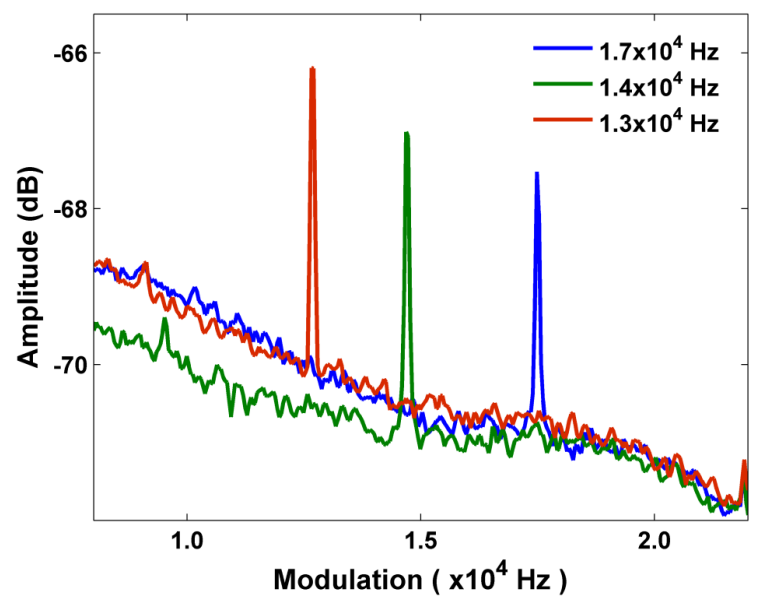

Fig. 6 Spectrum curve of LGA signal recorded by the microphone. Peaks of acoustic waves can be distinguished significantly from their noise. At lower region, there is stronger noise. Thus the modulation is only effective at a higher frequency

\section{Subsurface Corrosion Imaging}

Fig. 7 shows the object of the research which is made of carbon steel plate of $1.41 \mathrm{~mm}$ thickness, a mass of 6.21 gram and by a size of $29 \times 18 \mathrm{~mm}$. The scanning process is done on 
the top surface of the object while the corrosion is at the bottom. Corrosion is purposely created across the middle of the object by size $1 \times 18 \mathrm{~mm}$ (white line), while the size of scanning area is $10 \times 5 \mathrm{~mm}$ (yellow line) across the surface perpendicular to the corrosion. Scanning result of this area is used to construct an LGA image.

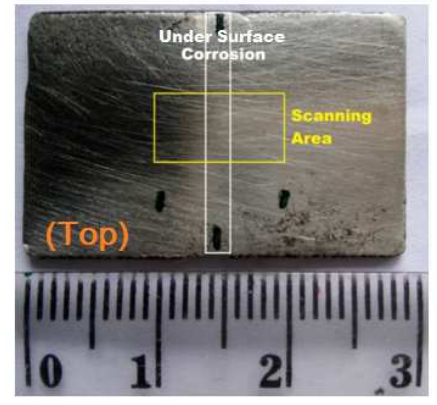

(a)

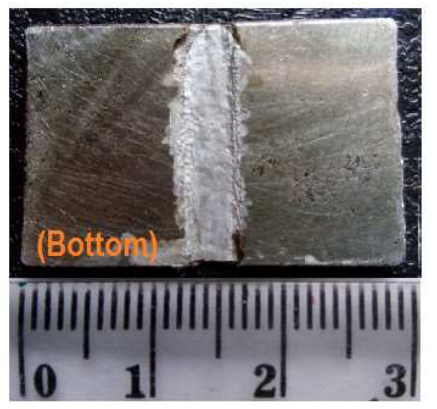

(b)
Fig. 7 (a) Research Object is made of a carbon steel plate in size of $29 \times 18 \mathrm{~mm}$ and thickness of $1.41 \mathrm{~mm}$. The scanning area is on the upper surface (Top) while corrosion is made underneath it (Bottom). Scanning area (yellow line) is done across the corrosion area (white line). (b) Corrosion on the object after 19.5 hours

After initial scanning, the object is submerged in a corrosive solution in 6 different amount of time as shown in Table 1 . The processing time taken into consideration is only immersion stage, while both scanning and measuring period are negligible. The amount of time for submerging the object in the solution is used as a reference for corrosion period. The object is weighed to measure the change of mass. Micrometre screw gauge is used to measure the depth of crevice to the end tip.

TABLE I

MASS AND DEPTH CHANGE DURING CORROSION

\begin{tabular}{|c|c|c|c|}
\hline No. & $\begin{array}{c}\text { Immersion time } \\
\text { (Hour) }\end{array}$ & $\begin{array}{c}\text { Mass } \\
(\mathbf{g r})\end{array}$ & $\begin{array}{c}\text { Corrosion depth } \\
(\mathbf{m m})\end{array}$ \\
\hline 1. & 0 & 6.21 & 0 \\
\hline 2. & 5 & 6.08 & 0.23 \\
\hline 3. & 7.5 & 6.05 & 0.29 \\
\hline 4. & 11 & 5.94 & 0.47 \\
\hline 5. & 12 & 5.91 & 0.52 \\
\hline 6. & 15 & 5.85 & 0.62 \\
\hline 7. & 19.5 & 5.75 & 0.73 \\
\hline
\end{tabular}

LGA image of the research object before corrosion process is shown in Fig. 8 which shows that there is no significant change in the intensity of the waves. This because the acoustic transmission coefficient is homogenous over the object surface before crevice corrosion is formed. More detailed evaluation is done by observing subsequent images of the surface in the next figures.

Stage by stage the object is introduced to corrosion and scanned to create LGA images (Fig. 9). First five hours after immersion, the image started to show intensity changes in the middle part. Fig. 9 shows a slight change of image on the corrosion area. Observation of the image explains increasingly significant difference after 7.5, 11 and 12 hours of corrosion. Corrosion starts from the middle part exposed to the corrosive solution. The next stage, after 15 and 19.5 hours, there is a significant increase of acoustic wave intensity. Referring to the depth of the corrosion in Table 1, the crevice is not only deeper but also starts spreading sideways. These substantial changes produced an LGA image with strong intensity, particularly after 19.5 hours. At this stage, the crevice has passed $50 \%$ of the object's thickness which is sufficient for a detailed analysis.

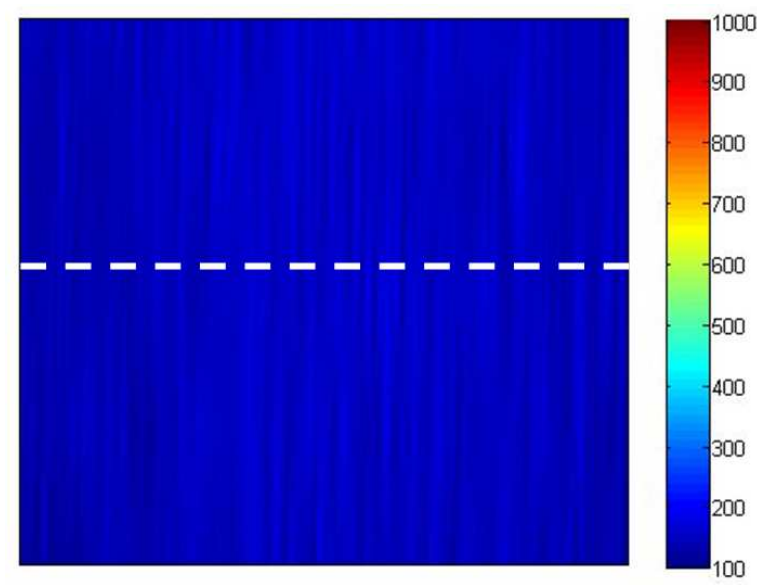

Fig. 8 LGA image of the object before corrosion shows an even distribution of wave intensity over the surface. The line-profile sample for the analysis is taken from the middle part of the image (dashed line)

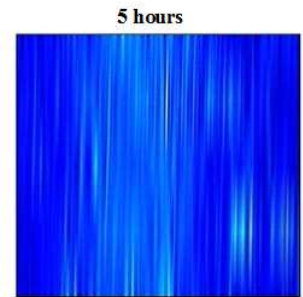

11 hours

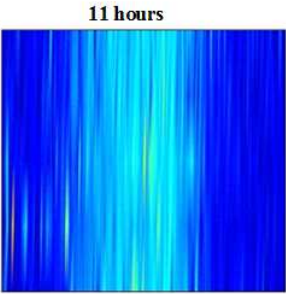

15 hours

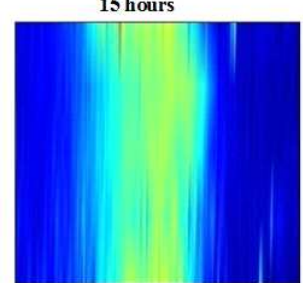

7.5 hours

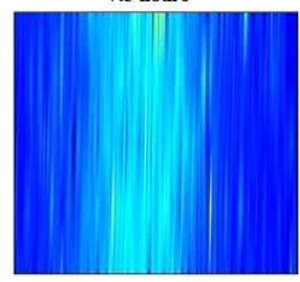

12 hours

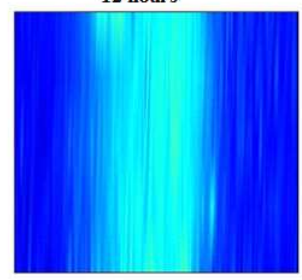

19.5 hours

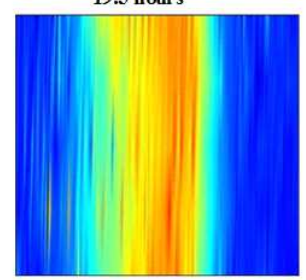

Fig. 9 Subsurface image of the object after 19.5 hours of immersion. A pit is formed in the exposed area as a result of mass destruction

Fig. 9 shows evolution process of subsurface corrosion for the first 5 hours to 19.5 hours when the depth of corrosion passed $50 \%$ of the object's thickness. Direct observation of the LGA images illustrates how the intensity of the image changes according to the growth of crevice corrosion under the surface of the object.

LGA images have shown clearly the development of subsurface crevice corrosion; however, for a better result, a line-profile analysis is done on mass change and depth of corrosion. A cross-section line is taken from the middle part of scanning area shown as a dashed line on Fig. 8. The lineprofile curves are combined as shown in Fig. 10 which 
illustrates how corrosion process increases the intensity of acoustic waves due to the length of immersion time. Moreover, the curves indicate the possibility to observe the development of corrosion earlier than five hours. This opportunity rises as there is a significant difference of the peaks between time 0 and 5 hours. Therefore, in this interval time, there is a chance of detecting corrosion at an early stage. Besides predicting the beginning of corrosion, the line-profile curve on Fig. 10 also shows the propagation of pit corrosion which can be seen from the expanding curve at the bottom along with the immersion time.

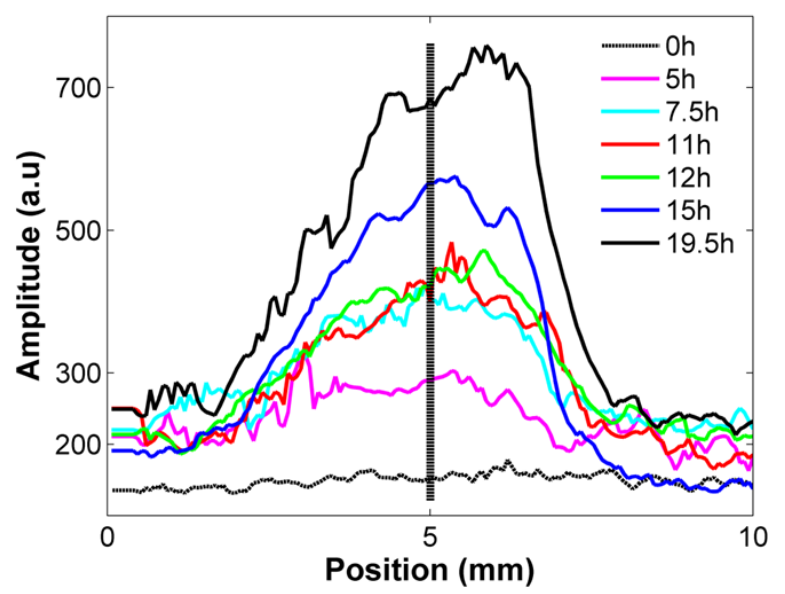

Fig. 10 The line-profile of LGA images shows the evolution process of subsurface corrosion. Besides increasing amplitude, the bottom or the curves are expanding as the immersion time increases. The vertical line is the position of line-profile for further analysis

Graph of immersion time to mass change and depth of crevice corrosion is shown in Fig. 11 to study the evolution process of crevice corrosion. The mass change to immersion time curve (left) shows a relatively constant gradient which represents the speed of corrosion in a unit of mass per unit of time. This linearity is assumed to occur because the increase in the exposed area is not strong enough to speed up the corrosion. Graph of immersion time to the depth of corrosion (right) has a different gradient to mass change. As the time of immersion increases, change of depth of corrosion is slower. Hypothetically, if the change of mass is constant, the change of depth of crevice is also constant. However, the result shows that this hypothesis is only true for a constant width of the crevice to time; the change of mass is affected only by the change of depth of crevice. In this case, the width changed over time which affects the reduction of mass during corrosion process. Therefore, the total lost mass per unit of time will be constant even though the depth change is slower which can be seen from curve trend shown in Fig. 11. Referring to Fig. 10, peaks and width of the crevice increases with the time of immersion. A rise of amplitude shows expanding area of which the transmission coefficient is changing as a result of corrosion.

An advanced analysis was done to study the acoustic transmission process on crevice corrosion by creating a transmission model on the three medium as shown in Fig. 12. LGA produced acoustic waves directly on the first medium, which is the carbon steel plate, with a modulation of $5 \times 10^{3} \mathrm{~Hz}$. Characteristic of this material is given by impedance of $45.4 \mathrm{Mrayl}$ and speed of $6000 \mathrm{~m} / \mathrm{s}$. The waves go through the second medium, crevice corrosion with thickness $\boldsymbol{L}$ filled with liquid, and thus the approach used for the medium is given by water characteristic with an impedance of 1.48 Mrayl and speed of wave $1480 \mathrm{~m} / \mathrm{s}$. After passing the crevice corrosion, acoustic signals reached the last medium and be recorded by the microphone. As the microphone tip is aluminium, the characteristic used in the model is the impedance of 17.4 Mrayl and speed of wave $6320 \mathrm{~m} / \mathrm{s}$.

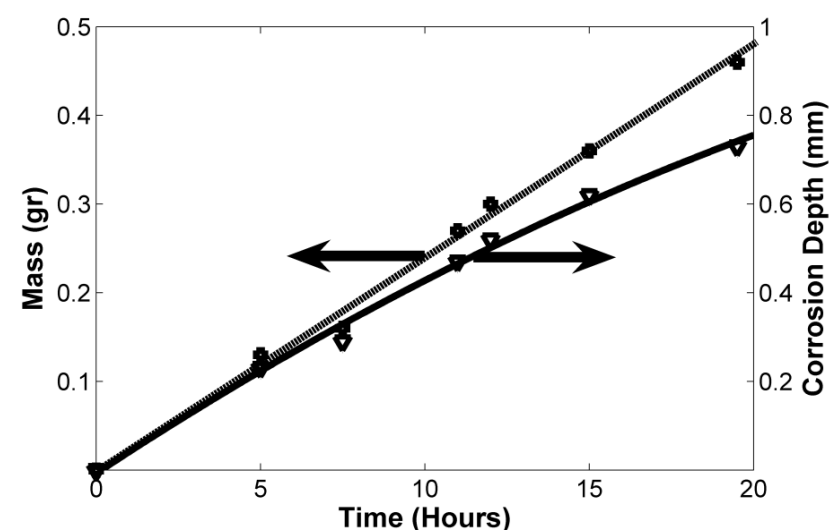

Fig. 11 Change of mass of the Object to time graph (Left) and corrosion depth to time graph (Right). The gradient of the change of mass graph tends to be constant, while the gradient of the corrosion depth graph is decreasing with time. These data represent detailed information of the evolution process of crevice corrosion

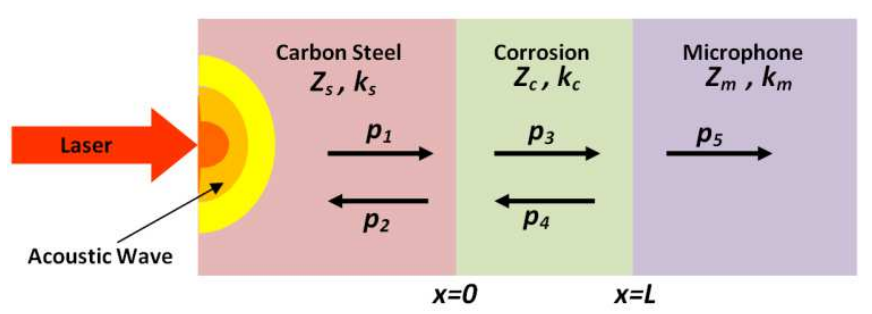

Fig. 12 Diagram of acoustic transmission model on three mediums; a carbon steel plate, a corrosion crevice, and a microphone. Each medium is characterised by different acoustic impedance $Z_{i}$, and wave number $k_{i}$. LGA will produce acoustic waves directly on the carbon steel plate which go through the crevice corrosion (with thickness $L$ ) and be detected by the microphone

Applying the scheme on Fig. 12, a complex wave equation can be formulated according to its parameter and geometrical properties. The position of the laser is set to be perpendicular to the surface so that the acoustic waves' propagation angle of incidence $\theta$ is $90^{\circ}$. Using the same resolution as of Equation 8, a set of wave transmission and reflection function can be derived as shown in Table 2 .

TABEL II

WAVE EQUATION SET SHOWn IN Fig. 12

\begin{tabular}{|c|c|c|}
\hline Carbon Steel & Corrosion & Microphone \\
\hline$p_{1}=P_{1} e^{j\left(\omega t-k_{s} x\right)}$ & $p_{3}=P_{3} e^{j\left(\omega t-k_{c} x\right)}$ & $p_{5}=P_{5} e^{j\left(\omega t-k_{m} x\right)}$ \\
\hline$p_{2}=P_{2} e^{j\left(\omega t+k_{s} x\right)}$ & $p_{4}=P_{4} e^{j\left(\omega t+k_{c} x\right)}$ & \\
\hline
\end{tabular}

Equations in Table 2 are used to formulate a transmission intensity coefficient equation for the model as follow 


$$
T_{I}=\frac{4}{2+\left(z_{m} / z_{s}+z_{s} / z_{m}\right) \cos ^{2} k_{c} L+\left(z_{c}^{2} / z_{s} z_{m}+z_{s} z_{m} / z_{c}^{2}\right) \sin ^{2} k_{c} L} .
$$

$Z_{i}$ and $k_{i}$ are acoustic impedance and wave number consecutively, for a medium with an index $i$ are $s$ (carbon steel), $c$ (corrosion) and $m$ (microphone). Curves of calculation result and of measurement are compared in Fig. 13 which shows both are well fitted. As for the shallower corrosion, there are fluctuations possibly caused by irregular crevice corrosion resulting in different transmission coefficient. The difference between the curves is calculated by a method of mean absolute percentage error (MAPE) [24], giving a result of $12.5 \%$.

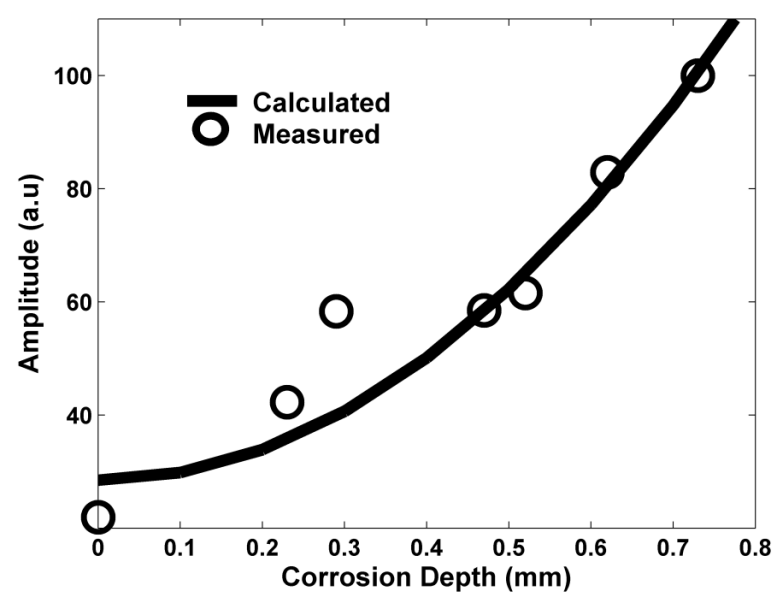

Fig. 13 Photoacoustic amplitude to corrosion depth graph, measurement result fits the calculation product using Equation 9.

\section{CONCLUSION}

LGA interaction process has proven to be useful to construct a detailed image of subsurface corrosion. The system built in this research was able to capture the evolution process of subsurface crevice corrosion from initiation to the resulting destruction stage. The LGA images produced can be used to study the geometrical formation of crevice corrosion and its relation to the mass degradation of the object. The mathematical model formulated can explain the physical process of corrosion with a mean absolute percentage error (MAPE) of $12.5 \%$.

\section{NOMENCLATURE}

$\mathrm{V}$
$\mathrm{p}$
$\mathrm{C}$
$\mathrm{v}$
$\mathrm{z}$
$\mathrm{k}$
$\mathrm{u}$
$\mathrm{R}$
$\mathrm{T}$

volume

pressure

thermal capacity

velocity

acoustic impedance

wavenumber

velocity component

reflectance coeff.

$\mathrm{T}$ transmittance coeff.

$\mathrm{m}^{3}$
$\mathrm{~Pa}$
$\mathrm{~J} / \mathrm{K}$
$\mathrm{m} / \mathrm{s}$
$\mathrm{rayl} / \mathrm{m}^{2}$
$\mathrm{~cm}^{-1}$
$\mathrm{~m} / \mathrm{s}$

Greek letters

$\kappa \quad$ isothermal compressibility

$\beta \quad$ volumetric thermal expansion

$\begin{array}{lll}\eta & \text { mass density } & \mathrm{kg} / \mathrm{m}^{3} \\ \theta & \text { angle } & \mathrm{rad} \\ \Phi & \text { temperature } & \mathrm{K}\end{array}$

wave function

\section{Subscripts \\ s sound \\ i incident \\ $r \quad$ reflected \\ t transmitted \\ I intensity \\ ext external}

\section{ACKNOWLEDGMENT}

This work was supported by Research Program of the Ministry of Research, Technology and Higher Education, Republic of Indonesia.

\section{REFERENCE}

[1] M.A. Fazal, A.S.M.A. Haseeb, and H.H. Masjuki, "Effect of Temperature on the Corrosion Behaviour of Mild Steel upon Exposure to Palm Biodiesel", Proceeding of the International Conference on Advanced Science, Engineering and Information Technology, 2011, pp.505-510.

[2] Nordin, Yahaya, Ahmed, and M.A. Budiea, "Near Shore Environment and Its Corrosiveness Towards Steel Pipelines", International Journal on Advanced Science, Engineering and Information Technology, Vol.2, pp.66-69, 2012.

[3] U.B. Halabe and R. Franklin, "Detection of Cracks in Long Painted or Rusted Steel", European International Journal of Science and Technology, Vol. 2 pp.161-182, 2013.

[4] A. Ajiz, Gunawarman, and J. Affi, "The Effects of Short-Time Solution Treatment and Short-Time Aging on Mechanical Properties of Ti-6Al-4V for Orthopaedic Applications", International Journal on Advanced Science, Engineering and Information Technology, Vol.5, pp.329-334, 2015.

[5] S. Suresh, M. Akmal Jamil, S. Sulaiman, and M.R.M. Shokor, "Optimization of Electrode Material for EDM Die-sinking of Titanium Alloy Grade 5 - Ti6Al4V”, International Journal on Advanced Science, Engineering and Information Technology, Vol.6, pp.534-539, 2016.

[6] G.G. Diamond, P. Kubasiak, K.P. Kyeyune, A.M. Wootton, and C. Bishop "Remote Detection of Corrosion under Paint (CUP) from Distance Greater than 5 metres", Proceeding in 11th European Conference on Non-Destructive Testing (ECNDT), 2014, pp.1-10.

[7] R.F. Anastasi and E.I. Madaras, "Terahertz NDE for Under Paint Corrosion Detection and Evaluation", Review of Quantitative Nondestructive Evaluation, Vol.25, pp.515-522, 2006.

[8] M.T. Ghasr, S. Kharkovsky, R. Zoughi, and R. Austin, "Comparison of Near-Field Millimeter-Wave Probes for Detecting Corrosion Precursor Pitting Under Paint", IEEE Transactions on Instrumentation and Measurement, Vol. 54, pp.1497-1504, 2005.

[9] W. Geary, "Analysis of A Corrosion Under Insulation Failure in A Carbon Steel Refinery Hydrocarbon Line", Case Studies in Engineering Failure Analysis, Vol.1, pp.249-256, 2013.

[10] N.N. Aung, W.K. Wai, and Y.J. Tan, "A Novel Electrochemical Method for Monitoring Corrosion Under Insulation", Anti-Corrosion Methods and Materials, Vol.53, pp.175-179, 2006.

[11] S.A. Majid and A. Balamesh., "Single Side Imaging of Corrosion Under Insulation Using Single Photon Gamma Backscattering", Research in Nondestructive Evaluation, Vol.25, pp.172-185, 2014.

[12] G.F. Kennell, R.W. Evitts, and K.L. Heppner, "A Critical Crevice Solution and IR Drop Crevice Corrosion Model", Corrosion Science, Vol.50, pp.1716-1725, 2008

[13] P.R. Roberge, Handbook of Corrosion Engineering, New York, USA: McGraw-Hill, 1999.

[14] Z. Sun, B. Rocha, K.T. Wu, and N. Mrad, "A Methodological Review of Piezoelectric Based Acoustic Wave Generation and Detection Techniques for Structural Health Monitoring", International Journal of Aerospace Engineering, Vol.2013, pp.1-22, 2013 . 
[15] T. Wang, S. Nandy, H.S. Salehi, P.D. Kumavor, and Q. Zhu, "A lowcost photoacoustic microscopy system with a laser diode excitation", Biomedical Optics Express, Vol. 5, pp.3053-3058, 2014.

[16] K. Maslov and L.V. Wang, "Photoacoustic imaging of biological tissue with intensity-modulated continuous-wave laser", Journal of Biomedical Optics, Vol.13, pp. 0240061-0240065, 2008.

[17] F. Gao, X. Feng, X. Miao, and Y. Zheng, "A Prototype for a Palmsized Photoacoustic Sensing Unit”, X Acoust. Imaging Sens., Vol.1, pp:73-77, 2015

[18] L.V. Wang, "Tutorial on Photoacoustic Microscopy and Computed Tomography", IEEE Journal of Selected Topics in Quantum Electronics, Vol. 14, pp.171-179, 2008.

[19] L.V. Wang, Photoacoustic Imaging and Spectroscopy, Boca Raton, USA: CRC Press; Taylor \& Fancis Group, 2009.
[20] L.Vocadlo, J.P. Poirer, and G.D. Price, "Grüneisen Parameters and Isothermal Equations of State", American Mineralogist Journal, Vol. 85, pp.01-06, 2000.

[21] L.E. Kinsler, A.R. Frey, A.B. Coppens, and J.V. Sanders, Fundamentals of Acoustics $4^{\text {th }}$ ed., New York, USA: John Wiley \& Sons, Inc. , 2000.

[22] K. Watanabe, Ed., Integral Transform Techniques for Green's Function, ser. Lecture Notes in Applied and Computational Mechanics, Switzerland: Springer, 2014, vol. 71.

[23] T. Starecki, "Ultra-low-noise preamplifier for condenser microphones", Review of Scientific Instruments, Vol.81, pp.1247021-1124702-4, 2010.

[24] J.S. Amstrong and F. Collopy, "Error measures for generalizing about forecasting methods: Empirical comparisons", International Journal of Forecasting, Vol. 08, pp.69-80, 1992. 